\title{
Development of optimal stimuli in a heterogeneous model of epileptic spike-wave oscillations
}

\author{
Nishant Sinha*, Peter N. Taylor ${ }^{\dagger}$, Justin Dauwels* and Justin Ruths ${ }^{\ddagger}$ \\ * School of Electrical \& Electronic Engineering, \\ Nanyang Technological University, Singapore. \\ Email: nsinha@ntu.edu.sg,jdauwels@ntu.edu.sg \\ ${ }^{\dagger}$ School of Computing Science, Newcastle University, \\ United Kingdom. Email: peter.taylor@ncl.ac.uk \\ $\ddagger$ Singapore University of Technology and Design, \\ Singapore. Email: justinruths@sutd.edu.sg
}

\begin{abstract}
Neural simulation has been widely suggested as an alternative therapy for the treatment of medically-intractable seizures. Appropriate targeting of control stimuli at selected cortical locations may lead to seizure abatement. Neural population models describe the macroscopic neural activity that can be clinically recorded by an electroencephalogram (EEG). These models provide a safer way to develop and test the effect of such simulation strategies. In this study, a heterogeneously connected neural field model has been used which can replicate spatio-temporal patterns commonly observed in the EEG during generalized seizures. Seizure abatement has been formulated as an optimal control problem and the pseudospectral method has been used to develop stimuli with anti-ictogenic properties. The minimum energy optimal stimuli, developed in this study have been shown to abate seizures simulated from the model. It has been demonstrated that the control stimuli are spatially variant due to the underlying heterogeneity of the neural dynamics. This study provides a novel approach for designing optimal stimuli for seizure abatement while taking into account the heterogeneous dynamics of the human brain. It also raises the possibility of finding the appropriate set of cortical locations which may be stimulated to achieve the anti-seizure effect.
\end{abstract}

\section{INTRODUCTION}

Epilepsy is a neurological disorder characterised by abnormal brain rhythms recorded at the macroscopic level of EEG electrodes typically during seizures. Epileptic seizures can limit patients' quality of life and can even be life-threatening, as patients can lose motor control at critical moments [1]. The electrographic epileptiform phenomena in the case of absence seizures, myoclonic seizures and complex partial seizures are often comprised of spike-wave discharges (SWD). While these spike wave discharges appear across many channels of the EEG, variations between recording electrodes can also be observed [2]. It has been proposed that these heterogeneities may be important for seizure genesis and hence should be incorporated into computational models of SWD seizures [3], [4], [5], [6].

In around one third of patients, uncontrolled seizures continue despite attempted pharmacological intervention with anti-convulsant drugs [7], [8]. The lack of efficacy of pharmacological treatments in a large group of patients with epilepsy, together with their severe adverse effects, underscores the need to develop novel therapies or delivery modalities. Brain stimulation via electrodes has been suggested as an alternative therapeutic treatment for epilepsy [9], [10], [11] and has had success in treating other neurological disorders including the motor symptoms of Parkinson's disease [12], [13]. However, how to design the optimal treatment strategies for epilepsy using stimulation methods has not been thoroughly studied.

In silico mathematical models of neural field dynamics provide a safer way to explore the effect of brain stimulations than in vivo experimentation. With appropriate parameters and connectivity inferred from the recordings of various neuroimaging modalities, models produce excitation waveforms that are characteristic of seizures. Several models of SWD have been proposed in literature to study mechanisms of SWD seizure genesis and dynamics [14], [15], [5], [2], [16], [17], [6]. However, these have not been used to investigate stimulation optimization incorporating known spatial heterogeneities.

In our previous work [18], we used a homogeneous (space independent) model of SWD and derived optimal stimuli to abate seizures. A separate study performs seizure abatement by shifting the model parameter using a PID-type feedback back controller [19]. However, feedback controllers are not necessarily a preferred solution in neuroscience as the stimulus is always "on". In contrast, we developed a minimal stimuli which is triggered to abate seizure only when it is detected. Generalized seizures, however involve widespread brain areas with distinctly heterogeneous EEG signals.

In this paper, we have extended our previous work [18] to incorporate heterogeneous cortico-cortical connectivity. Our model is presented with cortico-cortical connectivities inferred from the propagation of epileptic activity, shown in clinical analysis of human patients by [20]. To our knowledge, this is the first work investigating the heterogeneous (space variant) optimal control problem in a spatially extended heterogeneous epileptic neural population model of SWD. We present a systematic development of stimuli to abate seizures using techniques from optimal control theory.

\section{METHODS}

\section{A. Model}

Cortico-thalamic interactions, whereby the thalamus acts like a pacemaker and the cortex provides pathways for the genesis and maintenance of seizure have been enumerated 


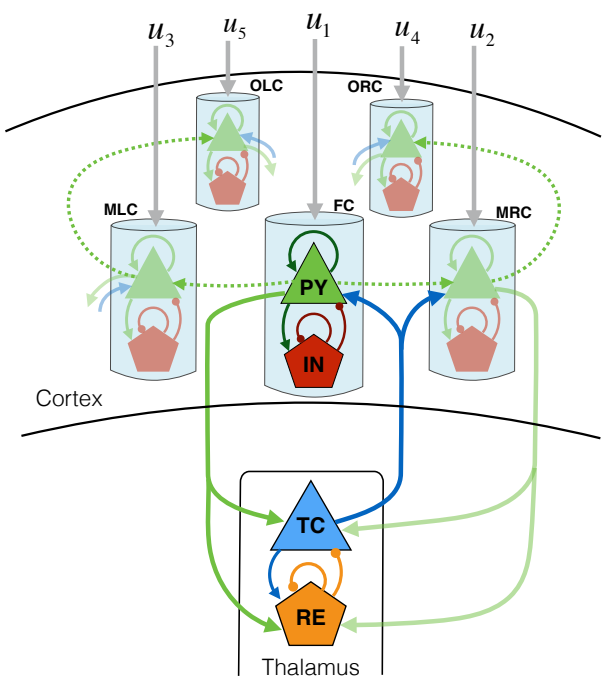

Fig. 1: Connectivity scheme of the model. Heterogeneous long range connectivity is shown between frontal cortex (FC), middle left cortex (MLC), middle right cortex (MRC), occipital left cortex (OLC) \& occipital right cortex (ORC).

by many recent studies [21], [22], [20]. Based on the Amari framework [23], we incorporate the knowledge of these structures in the two layer neural population model that we introduced in [6], [18], [24]. The cortical subsystem is composed of excitatory pyramidal (PY) and inhibitory interneuron (IN) populations. The thalamic subsystem includes variables representing populations of thalamocortical relay cells (TC) and neurons located in the reticular nucleus (RE).

The cortico-thalamic connections (shown by solid lines) are in agreement with the experimentally known connections [22]. As a first approximation, the heterogeneous long range cortico-cortical connectivity (shown by dotted lines) is inferred from the clinical findings of [20]. Based on the EEG-fMRI and DICS analysis, this study elicits the cortico-cortical network during generalized seizures and the propagation of pathological mechanism within this network. We use this heterogeneous cortico-cortical network because it permits a medium scale heterogeneous model for developing control stimuli to abate seizures. Following a similar approach, control stimuli can also be developed with more cortical areas, inferred either from DTI or any other neuroimaging modality. The model equations are given as follows:

$$
\begin{aligned}
d P Y_{i}(t)= & \tau_{1}\left(h_{p y}-P Y_{i}+\sum_{j} C_{1} A_{j i} f\left[P Y_{j}\right]\right. \\
& \left.-C_{3} f\left[I N_{i}\right]+C_{9} f[T C]\right)+u_{i} \\
d I N_{i}(t)= & \tau_{2}\left(h_{i n}-I N_{i}+C_{2} f\left[P Y_{i}\right]\right)+u_{i} \\
d T C(t)= & \tau_{3}\left(h_{t c}-T C-C_{6} f[R E]\right. \\
& \left.+C_{7} f[P Y]\right)+\alpha_{1} d W \\
d R E(t)= & \tau_{4}\left(h_{r e}-R E-C_{4} f[R E]\right. \\
& \left.+C_{5} f[T C]+C_{8} f[P Y]\right)
\end{aligned}
$$

for $i=1, \cdots, m$ with $m=5$ the number of cortical areas; $f[X]$ is the sigmoid function,

$$
f[X]=\left(1 /\left(1+\epsilon^{-X}\right)\right),
$$

and $X=P Y_{i}, I N_{i}, T C, R E$ with $\epsilon$ determines the sigmoid steepness; $\tau_{1 \ldots 4}$ and $h_{p y, i n, t c, r e}$ are the time constants and offset parameters respectively; $C_{1,2, \ldots 9}$ are the connections at the local level between $P Y_{i}, I N_{i}, T C, R E$ populations; $u_{i}$ is the input stimulus at location $i$. This follows the connection schematic shown in Fig. 1.

The model is excitable in the sense that upon the application of a sufficiently strong supra-threshold stimulus, a transient SWD will occur [6]. Noise can essentially cause a transient SWD since the noise is occasionally suprathreshold. This is incorporated in the model as $\alpha_{1} d W$, where $d W$ is the noise and $\alpha_{1}$ scales the amplitude. The parameter values used to simulate seizures from the model are $C=(1.8,4.0,1.5,0.2,10.0,1.5,3.0,3.0,1.0)$, $h_{p y}=(-1.0,-0.28,-0.29,-0.08,-0.08), \quad h_{\text {in }}=$ $(-3.4,-3.4,-3.4,-3.4,-3.4), h_{t c}=-2.1, h_{r e}=-5.0$, $\tau=(1.0,1.25,0.1,0.1)$ and $\varepsilon=250000$. These parameters are chosen such that the model is placed in the excitable state. The model solutions are computed numerically using a fixed step Euler-Maruyama solver in MATLAB.

\section{B. Optimal Control of Seizure Models}

Framing the seizure abatement goal as an optimal control problem facilitates leveraging the tools from control and optimization theory to provide a systematic approach to stimulus design for neuroscience. Typically, for most control objectives, there are many potential solutions; optimal control allows us to select from these many alternatives, the one solution that achieves the goal in an optimal (and unique) way.

Optimal control problems are formulated as optimization problems that impose a dynamic constraint, given by the differential equation that models the behavior of the system. In addition to the dynamic constraint, there are constraints that enforce the initial state of the system. The desired final state of the system can either be imposed as an additional constraint, or can be incorporated as part of the cost of the optimization problem.

During a seizure the system undergoes hypersynchronous oscillations, which are expressed in our model as approximately cyclic trajectories within the state space, where the state of the system is given by the vector $x(t)=$ $\left[P Y_{1}(t), \ldots, P Y_{m}(t), I N_{1}(t), \ldots, I N_{m}(t), T C(t), R E(t)\right]$.

We call the collection of points traced out by the state during such oscillations, the seizure manifold. The seizure abatement problem can then be described as driving the state $x(t)$ from a point on the seizure manifold to the non-excited, resting state of the dynamics. This resting state is given by the stable equilibrium at $x_{r}$. This goal, driving the system to $x_{r}$, can be achieved by including a constraint at the terminal time, similar to $x(T)=x_{r}$, or by including an error expression $\left(\left\|x(T)-x_{r}\right\|\right)$ in the cost of the optimization problem.

Mathematically, we formulate this optimal control problem 
with a cost in Bolza form as,

$$
\begin{array}{ll}
\min & \varphi(T, x(T))+\int_{0}^{T} \mathcal{L}(x(t), u(t)) d t, \\
\text { s.t. } & \dot{x}(t)=F(x(t), u(t)), \\
& e(x(0), x(T))=0, \\
& g(x(t), u(t)) \leq 0, \quad \forall t \in[0, T],
\end{array}
$$

where $x(t) \in \mathbb{R}^{2 m+2}$ and $u(t) \in \mathbb{R}^{m}$ is the state and control, respectively, at time $t \in[0, T] ; \varphi$ and $\mathcal{L}$ are the terminal and running costs; $F$ is the dynamics; and $e$ and $g$ are endpoint and path constraints, respectively.

While analytic methods exist for solving optimal control problems of this form, they typically do not scale well with the number of states and the complexity of the nonlinear dynamics. Therefore, we leverage computational techniques to solve problems of this size. The method employed in this study, the pseudospectral method, is a flexible interpolation method that discretizes the continuous optimal control problem into a nonlinear programming problem of the form,

$$
\begin{array}{ll}
\min & \varphi\left(T, \bar{x}_{N}\right)+\frac{T}{2} \sum_{i=0}^{N} \mathcal{L}\left(\bar{x}_{i}, \bar{u}_{i}\right) w_{i}, \\
\text { s.t. } & \sum_{k=0}^{N} D_{j k} \bar{x}_{k}=\frac{T}{2} F\left(x_{j}, u_{j}\right), \\
& e\left(\bar{x}_{0}, \bar{x}_{N}\right)=0, \\
& g\left(\bar{x}_{j}, \bar{u}_{j}\right) \leq 0, \quad \forall j \in\{0,1, \ldots, N\},
\end{array}
$$

where $\bar{x}_{j}=x\left(t_{j}\right)$ and $\bar{u}_{j}=u\left(t_{j}\right) ; t_{j}$ is the $j^{\text {th }}$ interpolation point; $w_{i}$ are weights characteristic of quadrature approximations of integrals; and $D$ is a constant coefficient matrix determined by the order of approximation/discretization $N$. Additional details regarding this method can be found in [25]. Nonlinear programming problems of this form can be solved by any number of commercial and open source solvers. In this work we use the commercial platform of AMPL and solver KNITRO ([26], [27]). When we formulate this problem, we also normalize the parameters so that they are all of comparable size. Here we rescale the time such that the optimizations converge more rapidly.

\section{RESULTS}

\section{A. Clinical EEG and model output}

Clinical EEG recordings of generalized seizures show typical spatio-temporal patterns across all electrode channels. As shown in Fig. 2(a), there is an abrupt transition of pathological spike wave discharges from seizure to non-seizure activity. The frequency also tends to decrease towards the end of the seizure. The pathological SWD rhythm consists of a fast spike and a slow wave which are typically of a much higher amplitude than the non-seizure EEG. Note that the temporal properties vary widely despite the increased correlation between channels during the seizure. For example, frontal channel (FC) usually records a higher amplitude than occipital channels (OLC and ORC). Furthermore, some channels show poly-SWD, while other show SWD. Therefore, the EEG activity between different electrode channels are not homogeneous, see e.g. [3] for further examples.

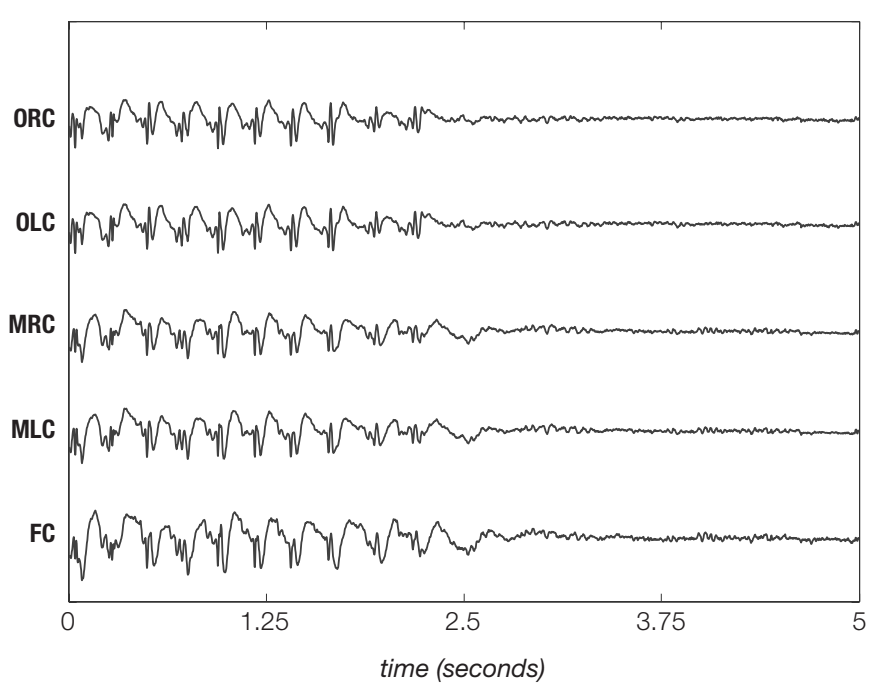

(a) Clinical EEG

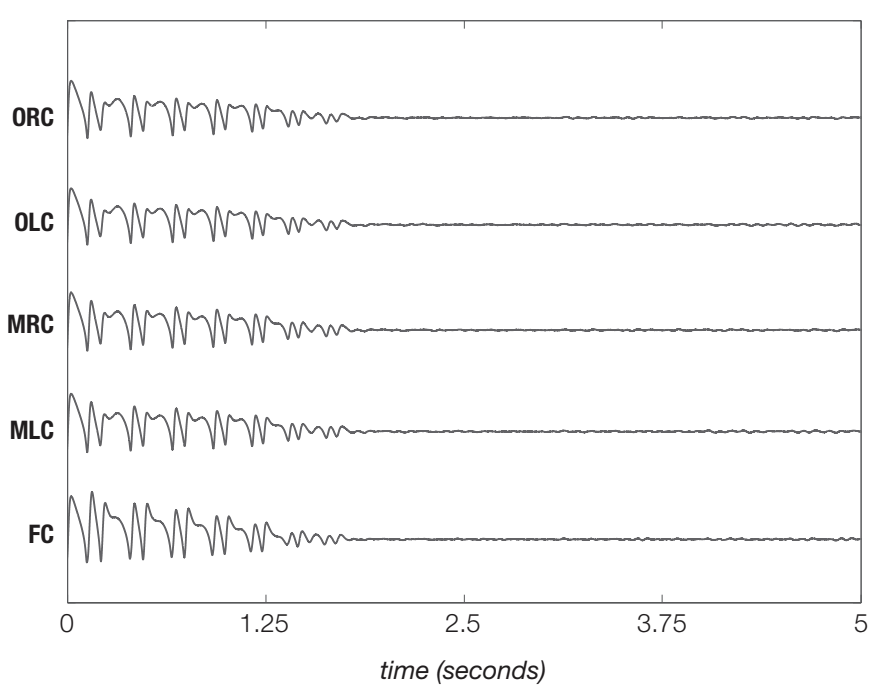

(b) Simulated EEG

Fig. 2: (a) Clinical EEG recording: Upper panel shows the EEG of a patient having idiopathic generalized epilepsy. (b) Simulated EEG: Lower panel shows the excitation of the PY variable when no control is applied to the frontal cortex (FC), middle left cortex (MLC), middle right cortex (MRC), occipital left cortex (OLC) and occipital right cortex (ORC)

Seizures simulated from the neural population model described in the previous section are shown in Fig. 2(b). The model directly enters into the seizure state if left uncontrolled, with all states starting with zero initial condition i.e., $x(0)=\mathbf{0}$. SWD, poly-SWD and slow wave oscillations can be observed in all simulated channels. The abrupt transition from the high amplitude seizure activity to non-seizure state is apparent. In spite of the synchrony during seizure, there is some degree of irregularity (heterogeneity) in the oscillations in both space and time with regard to waveform and amplitude. For example, the amplitude of FC is higher than those of ORC and OLC. Thus the model is capable of reproducing many key features observed in the clinical EEG. 


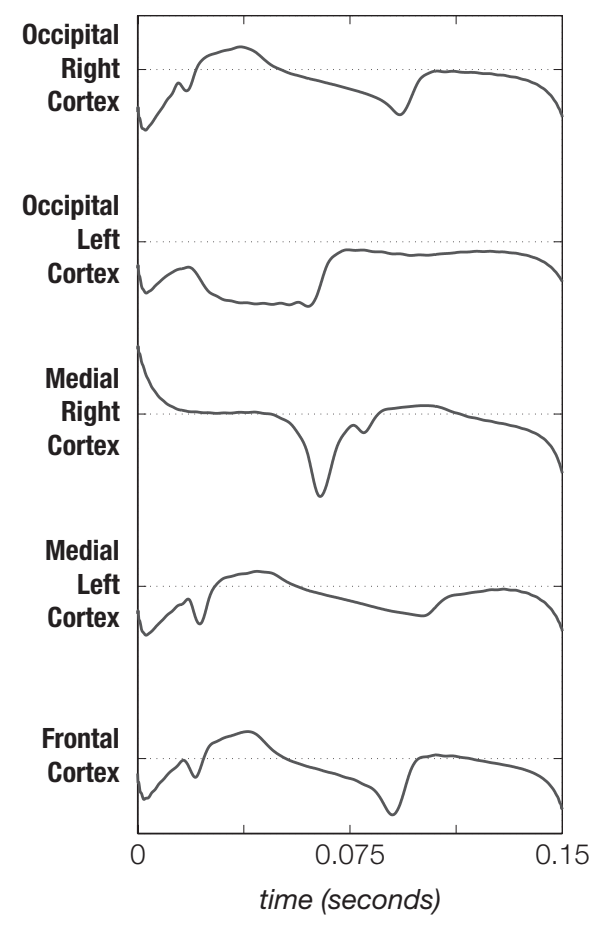

Fig. 3: The optimal control signals applied to the frontal cortex (FC), middle left cortex (MLC), middle right cortex (MRC), occipital left cortex (OLC) and occipital right cortex (ORC).

\section{B. Optimal Control}

We now apply the framework of optimal control theory to solve the seizure abatement problem. We formulate the optimal control problem with the goal of driving the system from the initial starting state $x(0)=x_{0}$ to the desired final state $x(T)=x_{r}$. Here we choose $x_{0}=\mathbf{0}$ because this will reliably generate a seizure, however, in principle we can enforce any arbitrary initial condition. The terminal state condition is given as the stable equilibrium point, $x_{r}$ and is enforced by a terminal constraint. Therefore, the endpoint constraints are given by $e(x(0), x(T))=\left[x(0)-x_{0}, x(T)-x_{r}\right]^{T}=\mathbf{0}$. The pseudospectral method also allows us to impose any arbitrary path constraint, in this case, we can bound the control amplitude $\left|u_{i}(t)\right| \leq A$, therefore, $g(x(t), u(t))=u(t)-A \leq \mathbf{0}$, where $A$ is the amplitude bound.

While solving this problem we design a set of bounded stimuli $u(t)$ that will drive the system from $x_{0}$ to $x_{r}$, as there are typically many, if not infinitely many, such stimuli that achieve this goal. We can impose a cost on this problem to select from these, the one input that does so in an optimal fashion. We chose a cost function of the form,

$$
\sum_{i=1}^{m} \int_{0}^{T} u_{i}^{2}(t) d t
$$

which minimizes the power (square integral) of the input signals. In physical systems, such control objectives tend to highlight inputs that are more physically relevant. In addition, for clinical systems, minimizing the input signals is important for the practical reason of being less invasive.
Using the pseudospectral method, we solve this optimal control problem for a fixed time interval of $T=0.15$ in real time units. This value is chosen as a realistic duration for the input to have effect on the system and so that there are not very many oscillations over the time interval because high frequency oscillations make the discretization problem more complex, requiring many more terms to keep the same level of accuracy. In practice this duration should be selected based on the period of the observed oscillation. The duration should be roughly similar between patients, although it can be tuned to achieve the best results based on the model. In principle it is possible to leave the time free as another decision variable, however, this increases the difficulty of the optimization. We discretize this time duration into $N=71$ segments, which corresponds to the order of approximation in the pseudospectral method. In practice the value of $N$ can be chosen large enough to capture the dynamics accurately. Due to the spectral characteristics of the method $N$ is typically substantially smaller than alternate (e.g., Runge-Kutta) methods.

The resulting minimum-power control inputs, in the sense that they minimize the cost function (1), are shown in Fig. 3. The trajectories of the PY excitation at the different cortical locations, following these input signals are plotted in Fig. 4. Compared with the uncontrolled PY trajectories in Fig. 2(b), the seizure is eliminated once the control signal completes at $T=0.15$. The trajectories are aggregated in the inset, zoomed axis to show the the evolution more clearly.

The power of the control signal in the occipital left cortex (OLC) is higher as compared to other regions which is shown in Fig. 5. However, in spite of different temporal evolution, it is evident that the control power of the frontal cortex (FC), middle left cortex (MLC), middle right cortex (MRC) and occipital right cortex (ORC) are approximately the same.

\section{CONCLUSION}

This pilot study addresses three fundamental questions. First, is the stimulus to abate seizures, space variant, since the epilepsy dynamics are known to be space variant? Second, is it possible to compute minimal invasive optimal stimuli to abate seizures for such space variant dynamics? Third, what is the efficacy of such stimuli? To investigate these questions, the seizure abatement problem was formulated as an optimal control problem and the pseudospectral method was used to obtain optimal stimuli with anti-ictiogenic properties. Using this methodology, we showed that it is possible to design input signals to abate seizures in a spatially variant epilepsy model. Our future work looks towards more rigorous testing of the efficacy of these signals as well as reducing the impact required to achieve seizure abatement.

It has been suggested that investigation of the heterogeneous spatial aspects of SWD could lead to an improved understanding and more effective treatments [28]. In this contribution, as a first approximation of spatially extended structural connectivity, we have used a spatially extended model of thalamocortical interaction with five cortical compartments. The cortico-cortical network formed by these cortical compartments has been shown to assist in the propagation of pathological activity during generalised seizures [20]. We 


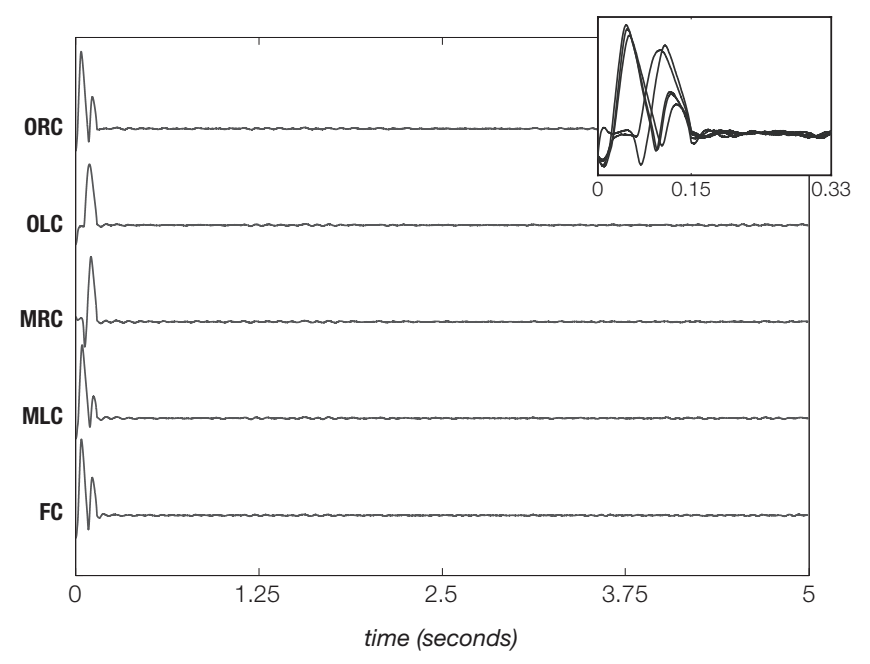

Fig. 4: The excitation of the PY variable when the optimal controls in Fig. 3 are applied for the frontal cortex (FC), middle left cortex (MLC), middle right cortex (MRC), occipital left cortex (OLC) and occipital right cortex (ORC).The inset, zoomed axis shows the same trajectories overlaid for the first 0.33 seconds, demonstrating the abatement of the seizure by the time the input signal finishes at $T=0.15$.

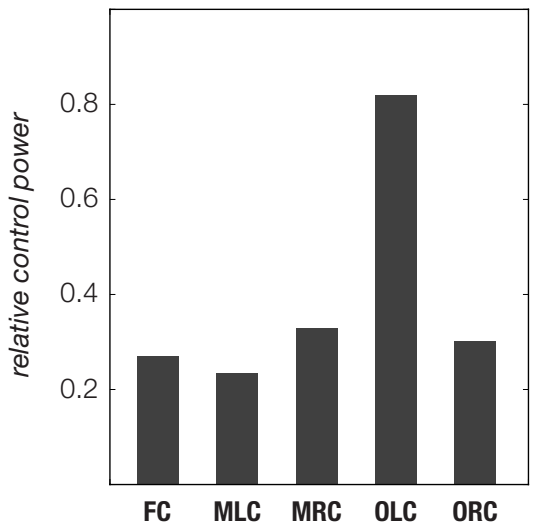

Fig. 5: The relative power of each applied control signal for the frontal cortex (FC), middle left cortex (MLC), middle right cortex (MRC), occipital left cortex (OLC) and occipital right cortex (ORC). Power is defined as the square integrated control signal over time.

have shown that this model can reproduce the waveform morphology of clinically observed SWD, spatio-temporally. From the seizures simulated from this model, we developed minimum energy optimal stimuli which could abate the seizure once it is detected. It was found that the optimal stimuli were spatially variant having different temporal evolutions for each of the cortical locations.

The relative power of the control stimulus was found to be similar for most of the regions except for a few, where it was found to be much higher (in this case, occipital left cortex (OLC)). This is suggestive of the fact that some cortical regions are more likely to induce seizures than others and hence require a higher power anti-ictogenic stimulus. In this regard, a very relevant question that this study raises is: what are the least possible locations that are required to be simulated to abate seizures from the entire corticothalamic network? This question is currently under investigation but this study serves as the first important step towards achieving this goal.

In future, to develop patient specific stimuli for seizure abatement, we aim to use patient specific connectivity. Many different approaches can be used based on either inferred functional connectivity (e.g. correlation, mutual information, phase coherence etc) or structural connectivity (e.g. diffusion weighted magnetic resonance imaging). Moreover, with the inclusion of multiple cortical compartments we aim to investigate the optimal location which should be simulated to abate seizures. Ultimately, it is crucial that both mathematical and experimental models are developed together so that the control stimuli developed in silico can be tested in vivo.

\section{REFERENCES}

[1] C. Selai, D. Bannister, and M. Trimble, "Antiepileptic drugs and the regulation of mood and quality of life (qol): the evidence from epilepsy," Epilepsia, vol. 46, no. s4, pp. 50-57, 2005.

[2] P. N. Taylor and G. Baier, "A spatially extended model for macroscopic spike-wave discharges," Journal of computational neuroscience, vol. 31, no. 3, pp. 679-684, 2011.

[3] G. Baier, M. Goodfellow, P. N. Taylor, Y. Wang, and D. J. Garry, "The importance of modeling epileptic seizure dynamics as spatio-temporal patterns," Frontiers in physiology, vol. 3, 2012.

[4] H. K. Meeren, J. P. M. Pijn, E. L. Van Luijtelaar, A. M. Coenen, and F. H. L. da Silva, "Cortical focus drives widespread corticothalamic networks during spontaneous absence seizures in rats," The Journal of neuroscience, vol. 22, no. 4, pp. 1480-1495, 2002.

[5] M. Goodfellow, K. Schindler, and G. Baier, "Intermittent spike-wave dynamics in a heterogeneous, spatially extended neural mass model," NeuroImage, vol. 55, no. 3, pp. 920-932, 2011.

[6] P. N. Taylor, G. Baier, S. S. Cash, J. Dauwels, J.-J. Slotine, and Y. Wang, "A model of stimulus induced epileptic spike-wave discharges," in Computational Intelligence, Cognitive Algorithms, Mind, and Brain (CCMB), 2013 IEEE Symposium on. IEEE, 2013, pp. 53-59.

[7] T. Keränen, M. Sillanpää, and P. J. Riekkinen, "Distribution of seizure types in an epileptic population," Epilepsia, vol. 29, no. 1, pp. 1-7, 1988.

[8] G. A. Baker, A. Jacoby, D. Buck, C. Stalgis, and D. Monnet, "Quality of life of people with epilepsy: a european study," Epilepsia, vol. 38, no. 3, pp. 353-362, 1997.

[9] S.-F. Liang, F.-Z. Shaw, C.-P. Young, D.-W. Chang, and Y.-C. Liao, "A closed-loop brain computer interface for real-time seizure detection and control," in Engineering in Medicine and Biology Society (EMBC), 2010 Annual International Conference of the IEEE. IEEE, 2010, pp. 4950-4953.

[10] S.-F. Liang, F.-Z. Shaw, D.-W. Chang, C.-P. Young, Y. L. Wang, and S. Y. Wu, "Live demonstration: A portable closed-loop seizure controller in freely moving rats," in Biomedical Circuits and Systems Conference (BioCAS), 2012 IEEE. IEEE, 2012, pp. 88-88.

[11] S. Saillet, S. Gharbi, G. Charvet, C. Deransart, R. Guillemaud, A. Depaulis, and O. David, "Neural adaptation to responsive stimulation: A comparison of auditory and deep brain stimulation in a rat model of absence epilepsy," Brain stimulation, vol. 6, no. 3, pp. 241-247, 2013.

[12] P. Limousin, P. Krack, P. Pollak, A. Benazzouz, C. Ardouin, D. Hoffmann, and A.-L. Benabid, "Electrical stimulation of the subthalamic nucleus in advanced parkinson's disease," New England Journal of Medicine, vol. 339, no. 16, pp. 1105-1111, 1998.

[13] X.-J. Feng, E. Shea-Brown, B. Greenwald, R. Kosut, and H. Rabitz, "Optimal deep brain stimulation of the subthalamic nucleus?a computational study," Journal of computational neuroscience, vol. 23, no. 3, pp. 265-282, 2007. 
[14] M. Breakspear, J. Roberts, J. R. Terry, S. Rodrigues, N. Mahant, and P. Robinson, "A unifying explanation of primary generalized seizures through nonlinear brain modeling and bifurcation analysis," Cerebral Cortex, vol. 16, no. 9, pp. 1296-1313, 2006.

[15] F. Marten, S. Rodrigues, P. Suffczynski, M. P. Richardson, and J. R. Terry, "Derivation and analysis of an ordinary differential equation mean-field model for studying clinically recorded epilepsy dynamics," Physical Review E, vol. 79, no. 2, p. 021911, 2009.

[16] Y. Wang, M. Goodfellow, P. N. Taylor, and G. Baier, "Phase space approach for modeling of epileptic dynamics," Physical Review E, vol. 85, no. 6, p. 061918, 2012.

[17] P. N. Taylor, M. Goodfellow, Y. Wang, and G. Baier, "Towards a large-scale model of patient-specific epileptic spike-wave discharges," Biological cybernetics, vol. 107, no. 1, pp. 83-94, 2013.

[18] J. Ruths, P. N. Taylor, and J. Dauwels, "Optimal control of an epileptic neural population model," Proceedings of the International Federation of Automatic Control, Cape Town, 2014.

[19] M. A. Kramer, B. A. Lopour, H. E. Kirsch, and A. J. Szeri, "Bifurcation control of a seizing human cortex," Physical Review E, vol. 73, no. 4, p. 041928, 2006.

[20] F. Moeller, M. Muthuraman, U. Stephani, G. Deuschl, J. Raethjen, and M. Siniatchkin, "Representation and propagation of epileptic activity in absences and generalized photoparoxysmal responses," Human brain mapping, vol. 34, no. 8, pp. 1896-1909, 2013.

[21] A. Destexhe, "Spike-and-wave oscillations based on the properties of gabab receptors," The Journal of neuroscience, vol. 18, no. 21, pp. 9099-9111, 1998.

[22] D. Pinault and T. J. O'Brien, "Cellular and network mechanisms of genetically-determined absence seizures," Thalamus \& related systems, vol. 3, no. 3, p. 181, 2005.

[23] S.-i. Amari, "Dynamics of pattern formation in lateral-inhibition type neural fields," Biological cybernetics, vol. 27, no. 2, pp. 77-87, 1977.

[24] P. N. Taylor, Y. Wang, J. Dauwels, and G. Baier, "Abatement of epileptic spike-wave discharges through single pulse stimulation," $B M C$ Neuroscience, vol. 14, no. Suppl 1, p. P13, 2013.

[25] J.-S. Li, J. Ruths, T.-Y. Yu, H. Arthanari, and G. Wagner, "Optimal pulse design in quantum control: A unified computational method," Proceedings of the National Academy of Sciences, vol. 108, no. 5, pp. 1879-1884, 2011.

[26] R. Fourer, D. M. Gay, and B. W. Kernighan, "AMPL: A mathematical programming language," 2002.

[27] R. H. Byrd, J. Nocedal, and R. A. Waltz, "Knitro: An Integrated Package for Nonlinear Optimization - Springer," Large-scale nonlinear optimization, 2006.

[28] H. Blumenfeld, "Cellular and network mechanisms of spike-wave seizures," Epilepsia, vol. 46, no. s9, pp. 21-33, 2005. 